\title{
Next-generation sequencing identified novel Desmoplakin frame-shift variant in patients with Arrhythmogenic cardiomyopathy
}

Xiaoping Lin ${ }^{1 \dagger}$, Yuankun Ma ${ }^{1 \dagger}$, Zhejun Cai ${ }^{1}$, Qiyuan Wang ${ }^{2}$, Lihua Wang ${ }^{2}$, Zhaoxia Huo ${ }^{3}$, Dan Hu4, Jian'an Wang ${ }^{1,5}$ and Meixiang Xiang ${ }^{1,5^{*}}$ (i)

\begin{abstract}
Background: Arrhythmogenic cardiomyopathy (AC) is one of the leading causes for sudden cardiac death (SCD). Recent studies have identified mutations in cardiac desmosomes as key players in the pathogenesis of AC. However, the specific etiology in individual families remains largely unknown.

Methods: A 4-generation family presenting with syncope, lethal ventricular arrhythmia and SCD was recruited. Targeted next generation sequencing (NGS) was performed and validated by Sanger sequencing. Plasmids containing the mutation and wild type (WT) were constructed. Real-time PCR, western-blot and immunofluorescence were performed to detect the functional change due to the mutation.

Results: The proband, a 56-year-old female, presented with recurrent palpitations and syncope. An ICD was implanted due to her family history of SCD/ aborted SCD. NGS revealed a novel heterozygous frame-shift variant (c.832de/G) in Desmoplakin (DSP) among 5 family members. The variant led to frame-shift and premature termination, producing a truncated protein. Cardiac magnetic resonance (CMR) of the family members carrying the same variant shown myocardium thinning and fatty infiltration in the right ventricular, positive bi-ventricular late gadolinium enhancement and severe RV dysfunction, fulfilling the diagnostic criteria of AC. HEK293T cells transfected with mutant plasmids expressed truncated DSP mRNA and protein, upregulation of nuclear junction plakoglobin (JUP) and downregulation of $\beta$-catenin, when compared with WT.
\end{abstract}

Conclusion: We infer that the novel c.832delG variant in DSP was associated with AC in this family, likely through Wnt/ß-catenin signaling pathway.

Keywords: Arrhythmogenic cardiomyopathy, Next generation sequencing, Genetic variant, Desmoplakin

\section{Background}

Arrhythmogenic cardiomyopathy (AC), characterized by gradual myocardium loss and fibrofatty replacement predominately in the right ventricle [1], is one of the primary causes for life-threatening ventricular arrhythmia

\footnotetext{
* Correspondence: xiangmx@zju.edu.cn

${ }^{+}$Xiaoping Lin and Yuankun Ma contributed equally to this work.

'Department of Cardiology, the Second Affiliated Hospital, Zhejiang

University School of Medicine, 88 Jiefang Road, Hangzhou 310009, Zhejiang, China

${ }^{5}$ Provincial Key Lab of Cardiovascular Research, 88 Jiefang Road, Hangzhou 310009, Zhejiang, China

Full list of author information is available at the end of the article
}

and sudden cardiac death (SCD), particularly in young and athletes [2]. The clinical presentations vary, including palpitations, syncope, symptomatic ventricular tachycardia, right heart failure and SCD. Sometimes, SCD was the only manifestation in AC patients, posting tremendous challenges to the diagnosis post mortem [2, 3]. Diagnosis of $\mathrm{AC}$, according to the guideline proposed by the international task force [4], is mainly based on findings of electrophysiological, structural and histological features, family history and genetic testing, hence, for those SCD patients, their family screening is of utmost importance. The current treatments for AC are mostly 
supportive and palliative [5], aiming at alleviation of arrhythmic and heart failure symptom and prevention of SCD, and heart transplantation is the final solution for end-stage patients. However, reversal or a complete cure of the disease requires further in-depth understanding of its etiology and pathogenesis.

Known as genetically determined cardiomyopathy, AC is mainly inherited in an autosomal dominant pattern with genetic and phenotypic heterogeneity [6]. Genetic studies have identified mutations in 5 components of cardiac desmosomes as main etiology of AC [6], namely Plakophilin 2 (PKP2), Desmoplakin (DSP), Desmoglein 2 (DSG2), Desmocollin 2 (DSC2), and Junction plakoglobin (JUP). Genetic defects of above genes can be found in $40-60 \%$ of AC patients [4]. However, the specific etiology in individual case remains largely unknown. First identified in a recessive disorder of keratoderma, woolly hair, and $\mathrm{AC}$ with left ventricle predominance (Carvajal syndrome) [7], DSP mutations are responsible for nearly $2-12 \%$ of AC patients $[8$, 9]. Recent study interestingly found that the left ventricle predominance or bi-ventricle involved phenotypes were associated with DSP non-missense mutations [10], but the genotype-phenotype correlations remain uncertain due to small sample size and need to be further characterized in individual families as well as large sample cohorts. Recent studies also suggested mutations that impaired ion channel activities may be causal or modifier to AC [11, 12], however, their prevalence is unsure.

In the current study, the underlying genetic defects in a 4-generation family presenting syncope, life-threatening ventricular arrhythmia and SCD were explored using next generation high-throughput sequencing platform, and a novel frame-shift variant c.832delG in DSP was identified. Cardiac magnetic resonance (CMR) further reveled the diagnosis of $\mathrm{AC}$ on two asymptomatic family members carrying the identical DSP variant. Through cosegregation and genotype-phenotype association analysis, and functional study on HEK293T cells, we infer that the novel frame-shift variant DSP c.832delG was associated with $\mathrm{AC}$ in this family.

\section{Methods}

\section{Study subjects}

The study protocol conforms to the ethical guidelines of the 1975 Declaration of Helsinki and was approved by Institutional Review Board (IRB) at the Second Affiliated Hospital, Zhejiang University School of Medicine (2016087). Written informed consent was obtained from all participants. Ten out of total 31 family members in a 4generation SCD family were recruited in the current study. A complete clinical information including family history, medical history, physical examination, lab test, 12lead echocardiogram (ECG), 24-h Holter monitoring, transthoracic echocardiography and CMR were collected.

\section{DNA extraction, target region capture and next-}

\section{generation sequencing}

The proband was selected for next generation sequencing using a commercial capture array (Roche NimbleGen, WI, USA) covering the exons and 50 base pairs of adjacent introns of 1876 cardiovascular diseases associated genes, including inherited cardiomyopathy, arrhythmogenic diseases, congenital heart diseases, mitochondrial diseases, etc.

Genomic DNA was extracted from peripheral blood lymphocytes by standard procedures using Axygen ${ }^{\circ}$ AxyPrep $^{\mathrm{Tm}}-96$ Blood Genomic DNA Kit (Axygen, NY, United States). The DNA libraries were constructed and sequenced using the Illumina 2000 platform (Illumina, CA, United States), providing an average sequencing depth of $>100$-fold of targeted exons.

\section{Data filtering and bioinformatics analysis}

The screening algorithms for potential disease-causing variants were as follows. Initially, intronic and synonymous exonic variants were excluded. Secondly, matched population and in-house database minor allele frequencies (MAF) were used to rule out common variants, defined by MAF $>0.01$. MAF of 3 major SNP databases were compared: ExAc (http://exac.broadinstitute.org/), 1000 genomes (http://www.1000genomes.org/) and ESP6500 (http://evs.gs.washington.edu/EVS/). Thirdly, rare non- synonymous variants were examined with HGMD (http://www.hgmd.cf.ac.uk/ac/), OMIM (http:// www.omim.org/) and ClinVar databases (https://www. ncbi.nlm.nih.gov/clinvar/) and finally analyzed using 3 known prediction tools, namely PolyPhen-2 (http://genetics.bwh.harvard.edu/pph2/), SIFT (http://sift.jcvi.org/) and MutationTaster (http://www.mutationtaster.org/), and categorized according to the recommended guidelines of the American College of Medical Genetics and Genomics (ACMG) and the Association for Molecular Pathology [13]. Sanger sequencing was performed bidirectionally for the verification of $A K A P 9$ c.10714C $>G$, FLNC c.7778C > G, SYNE1 c.25954C>T and DSP c.832delG in all participants.

\section{Plasmids construction and site-directed mutagenesis} AICSDP-9:DSP-mEGFP was a gift from the Allen Institute for Cell Science (Addgene plasmid \# 87424; http:// n2t.net/addgene:87424; RRID:Addgene_87,424) [14]. In order to facilitate the observation following transfection of mutant plasmid, GFP were cleaved and inserted in between the promoter and DSP gene. The frame-shift mutation was introduced into a wild-type DSP clone using a QuikChange II XL Site-Directed Mutagenesis Kit (Stratagene, La Jolla, CA, USA). The clones were sequenced to confirm the desired mutation and to exclude any other sequence variations. 


\section{RT-PCR and real-time PCR}

HEK293T cells were transfected with either blank, wild type or mutant plasmids using lipofectamine 3000 (Invitrogen, MA, USA) according to the manufacturer's instructions. Total RNA was extracted from transfected cells using the Trizol reagent (Invitrogen, MA, USA). cDNA was synthesized using PrimeScript RT reagent Kit (Takara, Shiga, Japan). The resulting cDNA was subjected to real-time PCR using TB Green Premix Ex Taq kits (Takara, Shiga, Japan) on an Applied Biosystems 7500 Fast Real-Time PCR System (ABI, CA, USA). The primers named "N-terminal" detected the mRNA levels in the $\mathrm{N}$-terminal side of the DSP mutation site, and the primers named "C-terminal" detected the mRNA levels in the C-terminal side of the DSP mutation site. GAPDH was used as an endogenous control.

The sequences of primers were listed as follows:

N-terminal-F: 5' -GCAGGATGTACTATTCTCGGC-3', N-terminal-R: 5' -CCTGGATGGTGTTCTGGTTCT-3'; C-terminal-F: 5' -ACATCATTCAGGCCACGT-3'; C-terminal-R: 5' - CCAGTTGACTCATGCGTA-3'; GAPDH-F: 5'-CGCTCTCTGCTCCTCCTGTT-3'; GAPDH-R: 5' -CCATGGTGTCTGAGCGATGT-3' .

\section{Western blots}

$24 \mathrm{~h}$ after transfection, total cell extracts were lysed by RIPA lysis buffer. Nuclear and cytoplasmic extracts were separated using Nuclear and Cytoplasmic Protein Extraction Kit (Beyotime Biotechnology, Shanghai, China). Next, proteins were separated by sodium dodecyl sulfate polyacrylamide gel electrophoresis (SDS-PAGE) and transferred to polyvinylidene fuoride (PVDF) membranes. The membranes were blocked for $1 \mathrm{~h}$ in a blocking solution of $5 \%$ (w/v) non-fat milk in PBS containing 0.1\% (v/v) Tween-20 and incubated at $4{ }^{\circ} \mathrm{C}$ overnight with indicated primary antibodies. Primary antibodies included antibodies against JUP (1:1000, sc-8415, Santa Cruz Biotechnology, CA, USA), $\beta$-catenin (1:1000, ab6302, Abcam, Cambridge, UK), GFP (1:1000, AF1483, Beyotime Biotechnology), GAPDH (1: 5000, 3683S, Cell Signaling Technology, MA, USA), Lamin B1 (1:1000, ET1606-27, HuaBio antibodies, China). Excess primary antibodies were washed off, and then the membranes were incubated with secondary antibodies conjugated with horseradish peroxidase for $1 \mathrm{~h}$ at room temperature. The western blot bands were visualized were visualized using the enhanced chemiluminescence western blotting detection system (Bio-Rad, CA, USA).

\section{Immunofluorescence analysis}

Cells seeded on cover slips were fixed with $4 \%$ paraformaldehyde (PFA)/PBS, permeabilized in $0.5 \%(\mathrm{v} / \mathrm{v})$ Triton X-100 (Sigma-Aldrich, MO, USA) and blocked with 5\% $(\mathrm{w} / \mathrm{v}) \mathrm{BSA}$. Then the cells were incubated using the antibody mouse-anti-JUP (1:1000, sc-8415, Santa Cruz
Biotechnology) overnight at $4{ }^{\circ} \mathrm{C}$, followed by secondary antibodies anti-mouse Alexa Fluor 594 (1:200, Thermo Fisher, A-21203, CA, USA) incubation in 5\% BSA in PBS for $1 \mathrm{~h}$ at room temperature. Finally, coverslips were mounted on microscope slides using mounting medium contained with DAPI (H-1200, Vector, CA, USA). Images were acquired using a fluorescence microscope (Leica, IL, USA). Colocalization analysis between JUP and nuclear was performed by Coloc 2 ImageJ in random high-power fields. Pearson's correlation coefficient was used to represent the colocalization quantification, +1 for perfect correlation, 0 for no correlation, and -1 for perfect anti-correlation. Optical confocal microscopies of cells were obtained using Leica TCS SP8 (Leica Microsystems Inc).

\section{Statistical analysis}

Data were presented as the means \pm SEM of at least three independent experiments. Student $\mathrm{T}$ test was performed to evaluate differences of continuous variables between two groups. One-way ANOVA was used for comparison among three groups. $P$ values of less than 0.05 were considered statistically significant. Statistical calculations were carried out using GraphPad Prism 8.0.1.

\section{Results}

\section{Demographic and clinical features of family members}

The pedigree of the family was shown in Fig. 1b. The proband (III-1), a 56-year-old female, was admitted to our hospital due to ICD battery depletion. She presented with a history of recurrent palpitations and syncope for 10 years. An ICD was implanted when she was 49 years old due to a positive family history of SCD/aborted SCD. Since no discharge was detected upon ICD implantation and she remained asymptomatic, no medication was administrated. Her paternal grandmother (I-2), uncle (II-4), and cousin (III-16) died suddenly. Her youngest sister (III7) experienced 2 episodes of syncope in her 38 and 40 years old, and an ICD was implanted in her 40 years old following resuscitation from a VT/VF event. Six appropriate discharges were detected in the following 6 years, and a second ICD was replaced when she was 46 years old. She was generally asymptomatic with $\beta$-blocker. Ten out of 31 family members were available and recruited for subsequent clinical and genetic evaluations (Fig. 1b).

The complete clinical features of all available family members were summarized in Table 1. No obvious depolarization and repolarization or structural abnormalities were detected by either ECG or transthoracic echocardiography tests for all participants. Though III-3, III-5 and IV-3 were asymptomatic, CMR were performed due to their potential positive genotype. Myocardium thinning and fatty infiltration was detected in the right apical area in III-3 when cardiac function was preserved. However, other than 


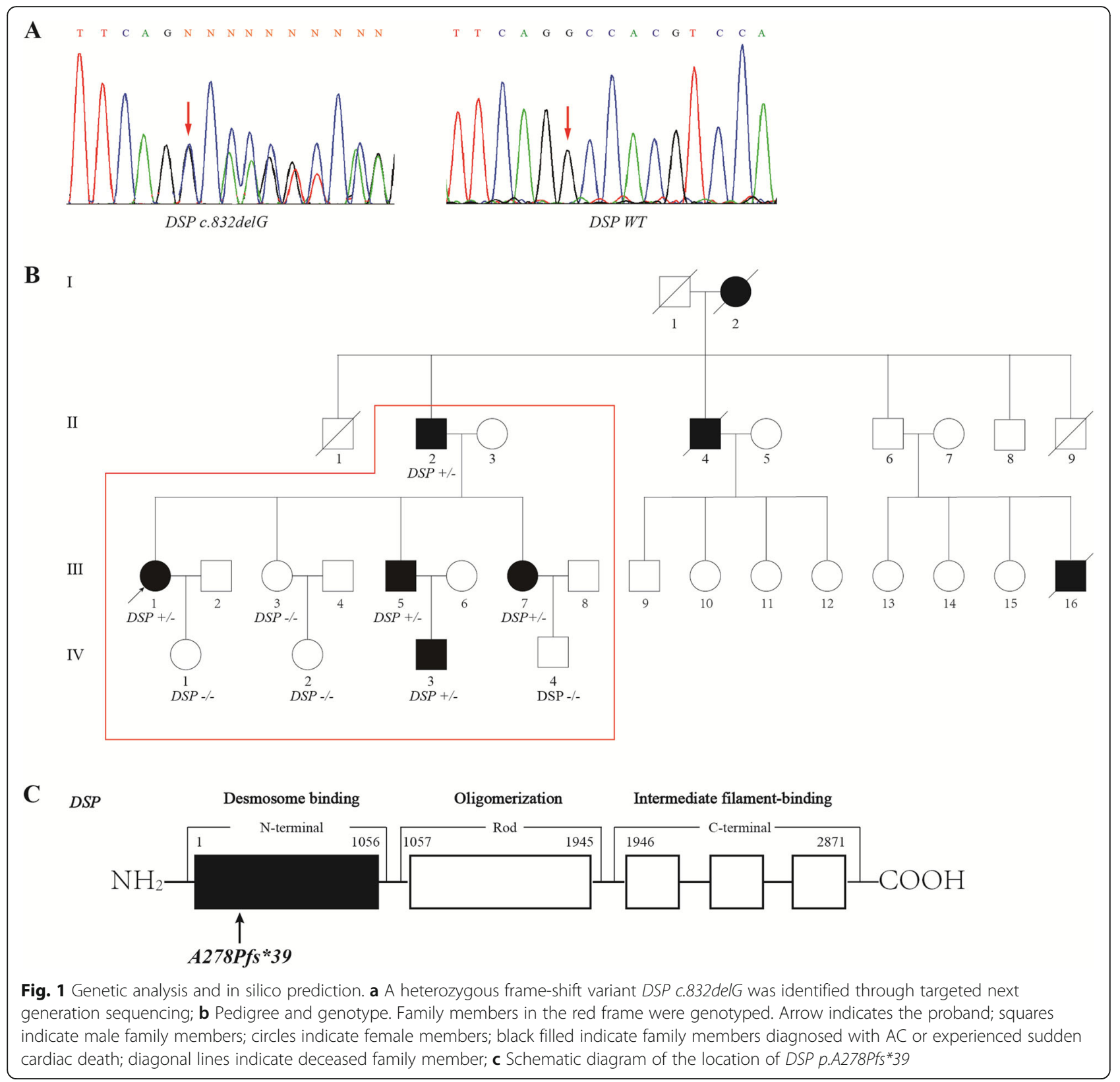

myocardium thinning and fatty infiltration in the right ventricle, positive bi-ventricular late gadolinium enhancement (LGE) and sever right ventricular dysfunction were detected in III-5 and IV-3. In addition, left ventricular function was moderately affected in IV-3 (Table 1 and Fig. 2). Thus, CMR manifestation of III-5 and IV-3 fulfilled the international Task Force criteria for the diagnosis of AC [4].

\section{Identification of pathogenic variant}

Next generation sequencing was performed on the proband. The average sequencing depths of sample on the targeted regions were 18,992-fold. More than $93.60 \%$ targeted regions were covered. We identified a total of 11 ,
583 variants in the proband, including 1232 nonsynonymous variants, 1494 synonymous variants, 8857 intronic variants and variants in un-translated regions (UTRs) (Additional file 1: Table S1). After filtering common ones, 82 non-synonymous variants distributed in 42 genes were left. Through screening of SCD associated genes, 4 novel heterozygous non-synonymous variants, including 2 missense variants, 1 non-sense variant and 1 frame-shift variant were selected for further in silico analysis (Table 2). Prediction tools yielded controversy results on A-kinase anchoring protein 9 (AKAP9) c.10714C > G and filamin $C$ (FLNC) $c .7778 C>G$, favoring them as harmless polymorphisms, thus their clinical significance 
Table 1 Clinical featuresand genotypes of family members

\begin{tabular}{|c|c|c|c|c|c|c|c|c|c|}
\hline No. & Gender & $\begin{array}{l}\text { Age } \\
\text { rangs } \\
(y)\end{array}$ & $\begin{array}{l}\text { Medical } \\
\text { history }\end{array}$ & ECG & Holter & $\mathrm{ECHO}$ & CMR & $\begin{array}{l}\mathrm{AC} \\
\text { Criteria }\end{array}$ & Genotype \\
\hline$\| 2$ & M & $80-85$ & $\begin{array}{l}\text { CHD, Dual- } \\
\text { chamber } \\
\text { pacemaker }\end{array}$ & $\begin{array}{l}\text { DDD pacing } \\
\text { rhythm, LBBB, } \\
\text { old } \mathrm{Ml}\end{array}$ & $\begin{array}{l}\text { DDD } \\
\text { pacing } \\
\text { rhythm }\end{array}$ & $\begin{array}{l}\text { LA } \\
\text { enlargement }\end{array}$ & - & - & $\begin{array}{l}\text { AKAP9+/ } \\
-F L N C \\
-/-D S P+/-\end{array}$ \\
\hline 113 & $\mathrm{~F}$ & $80-85$ & HTN & PVCs & $\begin{array}{l}\text { Frequent } \\
\text { PVCs, } \\
\text { PACs }\end{array}$ & Normal & - & - & $\begin{array}{l}\text { AKAP9 } \\
-/-F L N C+/ \\
-D S P-/-\end{array}$ \\
\hline III1 & $\mathrm{F}$ & $55-60$ & $\begin{array}{l}\text { Palpitation, } \\
\text { syncope, ICD }\end{array}$ & Normal & $\begin{array}{l}\text { Frequent } \\
\text { PVCs, } \\
\text { NSVT }\end{array}$ & Normal & - & Fulfilled & $\begin{array}{l}\text { AKAP9+/ } \\
-F L N C+/ \\
-D S P+/-\end{array}$ \\
\hline III3 & $\mathrm{F}$ & $50-55$ & Palpitation & PVCs & - & - & $\begin{array}{l}\text { RV apical myocardium thinning and fatty } \\
\text { infiltration }\end{array}$ & Unfulfilled & $\begin{array}{l}\text { AKAP9+/ } \\
-F L N C \\
-/-D S P-/-\end{array}$ \\
\hline III5 & M & $50-55$ & Asymptomatic & Normal & - & Normal & $\begin{array}{l}\text { RV myocardium thinningand myocardial } \\
\text { fatty infiltration, bi-ventricular LGE, } \\
\text { LVEF52\%, RVEF29\% }\end{array}$ & Fulfilled & $\begin{array}{l}\text { AKAP9 } \\
-/-F L N C \\
-/-D S P+/-\end{array}$ \\
\hline III7 & $\mathrm{F}$ & $45-50$ & $\begin{array}{l}\text { Syncope, VT/ } \\
\text { VF, ICD }\end{array}$ & $\begin{array}{l}\text { Low QRS } \\
\text { voltages }\end{array}$ & $\begin{array}{l}\text { Frequent } \\
\text { PVCs }\end{array}$ & Normal & - & Fulfilled & $\begin{array}{l}\text { AKAP9+/ } \\
-F L N C+/ \\
-D S P+/-\end{array}$ \\
\hline IV1 & $\mathrm{F}$ & $30-35$ & Asymptomatic & Normal & - & Normal & - & - & $\begin{array}{l}\text { AKAP9+/ } \\
-F L N C \\
-/-D S P-/-\end{array}$ \\
\hline IV2 & $\mathrm{F}$ & $25-30$ & Asymptomatic & PVCs & - & - & - & - & $\begin{array}{l}\text { AKAP9 } \\
-/-F L N C \\
-/-D S P-/-\end{array}$ \\
\hline IV3 & M & $20-25$ & Asymptomatic & PVCs & - & Normal & $\begin{array}{l}\text { RV myocardium thinningand myocardial } \\
\text { fatty infiltration, bi-ventricular LGE, } \\
\text { LVEF43\%, RVEF17\% }\end{array}$ & Fulfilled & $\begin{array}{l}\text { AKAP9 } \\
-/-F L N C \\
-/-D S P+/-\end{array}$ \\
\hline IV4 & M & $15-20$ & Asymptomatic & Normal & - & - & - & - & $\begin{array}{l}\text { AKAP9 } \\
-/-F L N C+/ \\
-D S P-/-\end{array}$ \\
\hline
\end{tabular}

ECG electrocardiogram; $E C H O$ echocardiogram; $C M R$ cardiac magnetic resonance; $F$ female; $M$ male; $C H D$ coronary artery disease; $H T N$ hypertension; $L B B B$ left bundle branch block; $M I$ myocardial infarction; $L A$ left atrium; $L V$ left ventricle; $R V$ right ventricle; $E F$ ejection fraction; $P A C S$ premature atrial contractions; $P V C S$ premature ventricular contractions; NSVT non-sustained ventricular tachycardia; $V T$ ventricular tachycardia; $V F$ ventricular fibrillation; ICD implantable cardioverter defibrillator

was uncertain. Though spectrin repeat containing nuclear envelope protein 1 (SYNE1) c.25954C > T non-sense variant was predicted to be disease-causing by MutationTaster, none of the family members presented with neuromuscular disorder as previously reported [15]. The DSP c.832delG (Fig. 1a) was predicted to be diseasecausing by MutationTaster, PolyPhen- 2 and SIFT. Sanger sequencing further revealed that the proband's father (I2 ), her two sisters (III-3 and III-7) and her daughter (IV-1) carried AKAP9 c.10714C > G. The proband's mother (I-3), her youngest sister (III-7) and her niece (IV-4) carried FLNC c.7778C > G. The proband's father (I-2), her youngest sister (III-7), her younger brother (III-5) and her nephew (IV-3) carried DSP c.832delG (Table 1). Hence only DSP c.832delG was co-segregated with positive phenotype in those characterized members of this family (Table 1; Fig. 1b), supporting the possible pathogenic role of this novel variant. According to ACMG criteria, AKAP9 c.10714C > G, FLNC c.7778C > G and SYNE1 c.25954C> $T$ variants were not co-segregated with positive phenotype in the current family (Table 1), hence, they were classified as benign strong 4 (BS4). However, the DSP c.832delG variant, as a frame-shift mutation, was well co-segregated with positive phenotype with in the family, thus was classified as pathogenic very strong (PVS1).

\section{DSP c.832delG led to truncated DSP mRNA and protein expression, increased JUP and decreased $\beta$-catenin expression in the nuclear}

The DSP c.832delG led to a frame shift and a premature termination codon (p.A278Pfs*39) (Fig. 1c), producing a truncated protein of 315 amino acids, compared with full-length of 2871 amino acids. Real-time PCR found that there were no significant differences between mutant and wild-type in mRNA levels in the $\mathrm{N}$-terminal side of DSP mutation, whereas, mRNA levels in Cterminal side of DSP mutation were only elevated in wild type cells (Fig. 3a-b), indicating the mRNA translation following the truncation was completely impaired. Western-bolt did not shown a difference of protein 


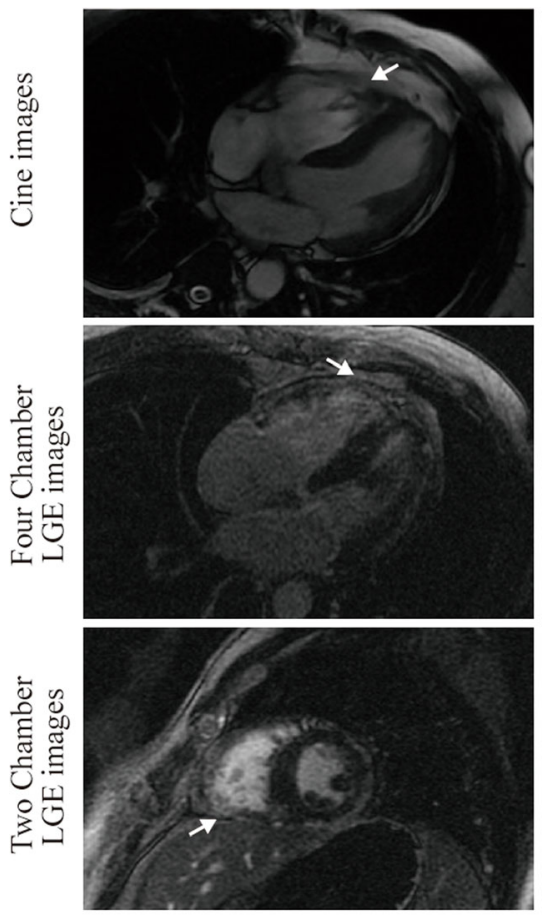

III5

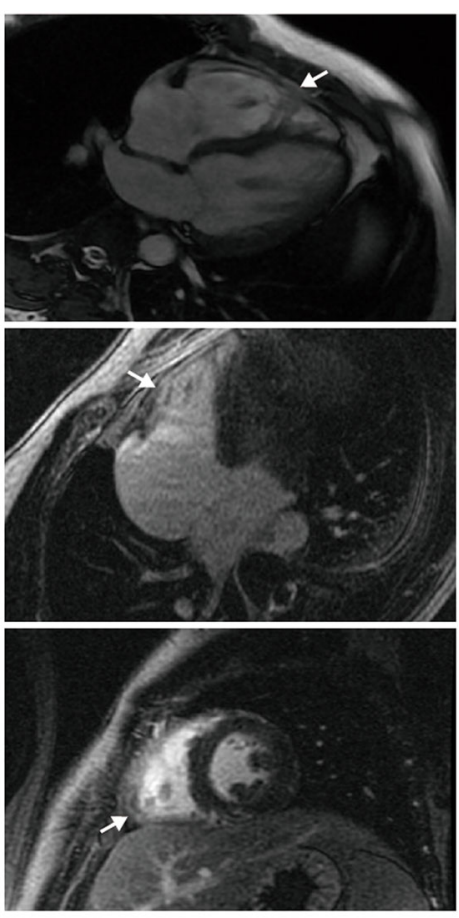

IV3

Fig. 2 Representative cardiac magnetic resonance images. Myocardium thinning and fatty infiltration (arrow) in the right ventricular and positive bi-ventricular late gadolinium enhancement were detected in III5 and IV3. Myocardium thinning and fatty infiltration (arrow) were detected in the right apical region in III-3. LGE, late gadolinium enhancement

expression between wild type and DSP c.832delG when using a DSP primary antibody, hence, protein truncation was examined using GFP antibody. Over-expression of plasmids carrying DSP c.832delG presented with significantly shortened protein, when compared with wild type (Fig. 3c), suggesting a truncating effect caused by the mutation. We then tested the down-stream protein change separately in cytoplasm and nuclear. DSP c.832delG over-expression led to upregulation of JUP and downregulation of $\beta$-catenin in the nuclear, without affecting their expression in the cytoplasm (Fig. 4a-c), when compared with wild type plasmids. Immunofluorescence through confocal microscopy confirmed the upregulation of nuclear JUP upon transfection of mutant type plasmids (Fig. 5a-c), indicating accumulation of nuclear JUP and suppression of $\mathrm{Wnt} / \beta$-catenin signaling pathway may play a key role in the pathogenesis of $\mathrm{AC}$ due to DSP c.832delG.

\section{Discussion}

In the current study, through targeted next generation sequencing platform covering a board rang of inherited cardiovascular disease genes, a novel frame-shift variant DSP c.832delG is identified in a large SCD family. CMR unveils the typical manifestations of myocardium thinning, fatty replacement and severely impaired heart function, particular in the right heart of the variant carriers, fulfilling the international Task Force criteria for the diagnosis of AC [4]. Functional study on HEK293t cells reveals truncation of DSP protein, down-regulation of JUP and up-regulation of $\beta$-catenin expression in

Table 2 In silico predictions of 4 novel non-synonymous variants

\begin{tabular}{lllllll}
\hline Gene & cDNA alteration & AA alteration & Effect & Mutation Taster & PolyPhen-2 & SIFT \\
\hline AKAP9 & $c .10714 C>G$ & p.P3572A & Missense & Polymorphism (0.99) & Benign 0.003 & Tolerate (0.86) \\
FLNC & $c .7778 C>G$ & p.T2593S & Missense & Disease causing (0.99) & Benign0.055 & Tolerate (0.25) \\
SYNE1 & $c .25954 C>T$ & p.R8652X & Nonsense & Disease causing (0.99 & Disease causing & Disease causing \\
DSP & $c .832$ delG & p.A278Pfs*39 & Frame-shift & Disease causing0.99 & Disease causing & Disease causing \\
\hline
\end{tabular}


A

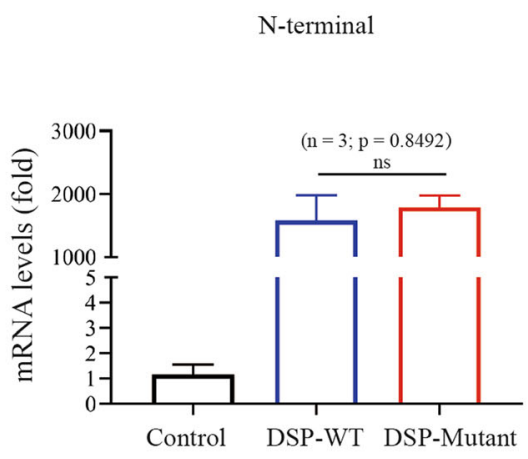

C

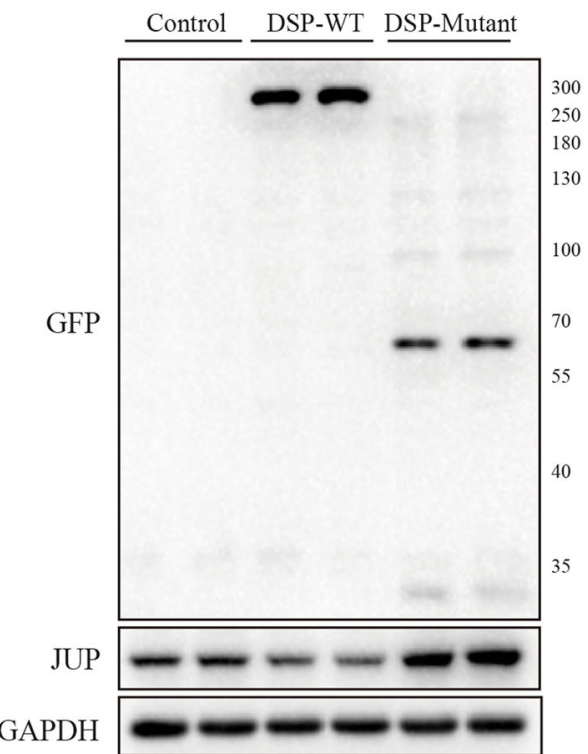

B

C-terminal

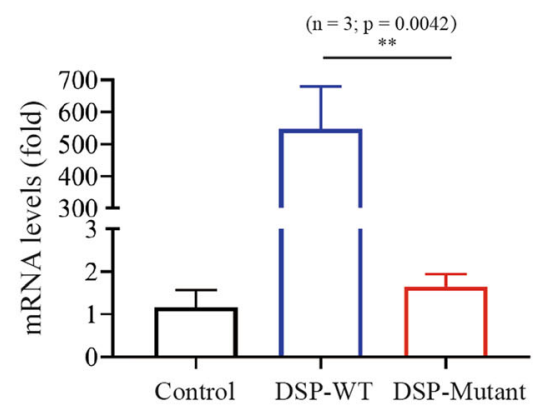

D

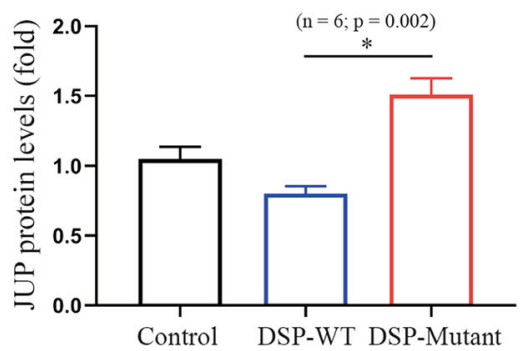

Fig. 3 DSP mRNA expression, total DSP and JUP protein expression. HEK293T cells were transfected with either blank, wild type or mutant plasmids. Blank plasmids without DSP gene served as control group. $\mathbf{a}-\mathbf{b}$ qPCR analysis for DSP mRNA levels in the N-terminal and C-terminal of the c.832delG mutation site. There were no significant differences between mutant and wild-type in mRNA levels in the N-terminal side of DSP mutation, whereas, mRNA level in C-terminal side of DSP mutation was only elevated in cells transfected with wild type plasmid transfection; c-d GFP antibody was used to exam the length of protein expressed in whole cell lysates. Mutant DSP protein was much shorter than wild type, suggesting truncation effect of the mutation. JUP expression was significantly increased in the mutant group. GAPDH served as an internal control. DSP, Desmoplakin; JUP, Junction plakoglobin; WT, wild type

nuclear, but not cytoplasm upon transfection of plasmids with DSP c.832delG.

Desmoplakin, a member of the plakin family, anchors other desmosome components to intermediate filaments as to maintain the integrity of desmosome structure [16]. SCD is reported to be more prevalent in DSP defect patients, especially truncations [17], when compared with other desmosome defects [9]. In our AC family, 4 family members present with $\mathrm{SCD} /$ aborted $\mathrm{SCD}$ as first clinical manifestation, and the VT/VF survivor carries DSP c.832delG truncation, consistent with previous findings. It has been proposed that DSP missense mutation exert a negative dominant effect whereas non-missense mutation exert haploinsufficiency [18], leading to phenotypic discrepancy. DSP missense mutation presents with more severe phenotype than non-missense mutation [19], such as earlier disease onset and more prevalence of lethal arrhythmia. However, this correlation is inconsistently reported in clinical studies. Up to date, the largest $\mathrm{AC}$ cohort with $D S P$ mutation recruiting 27 patients suggests that non-missense mutations is only associated with left-dominant forms [10]. In the current study, despite normal TTE, CMR exam sensitively detects that 2 of our DSP c.832delG carriers present mild to moderate left ventricle involvement, nevertheless, right ventricular impairment is dominant, suggesting phenotype is possibly mutation-dependent. Apparently, larger sample of AC cohort with various types of DSP mutation will be needed to further explore the genotype-phenotype correlation.

The canonical Wnt/ $\beta$-catenin signaling is considered to play a central role in the pathogenesis of AC with $D S P$ 


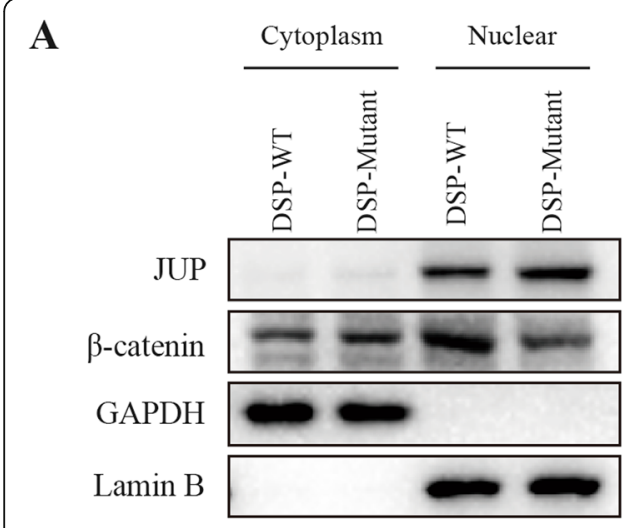

B

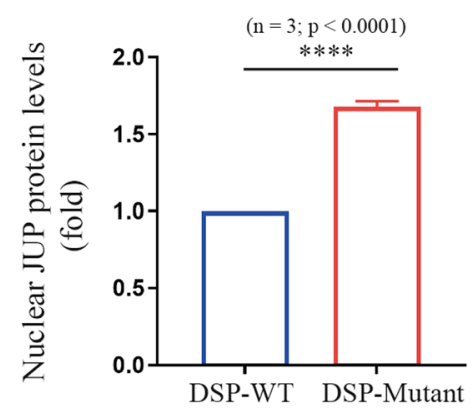

C

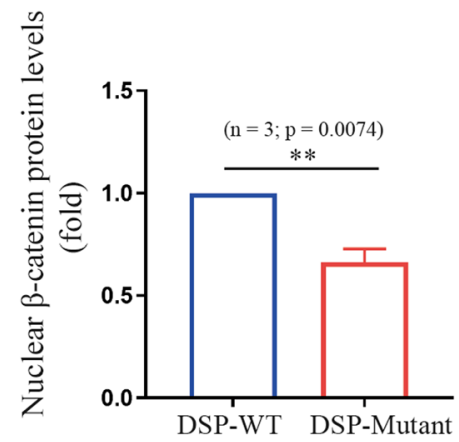

Fig. 4 JUP and $\beta$-catenin expression in cytoplasm and nuclear, separately. HEK293T cells were transfected with either wild type or mutant plasmids. $\mathbf{a}-\mathbf{b}$ JUP was significantly upregulated and $\mathbf{a}-\mathbf{c} \beta$-catenin was downregulated in cells transfected with mutant type in the nuclear, rather than cytoplasm, when compared with wild type DSP. GAPDH served as an internal control in the cytoplasm and Lamin B served as an internal control in the nuclear. Blank plasmids carrying no DSP gene served as control group. DSP, Desmoplakin; JUP, Junction plakoglobin; WT, wild type

A
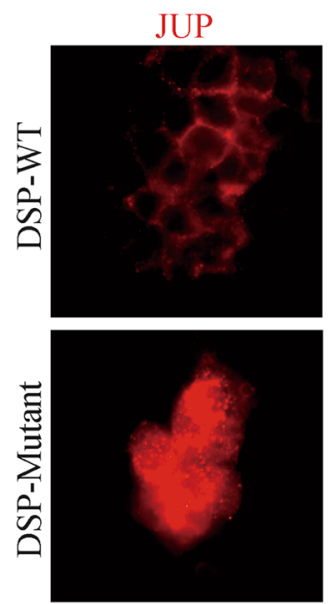

B
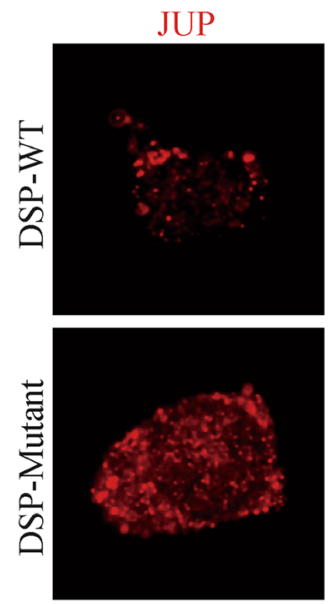

DAPI
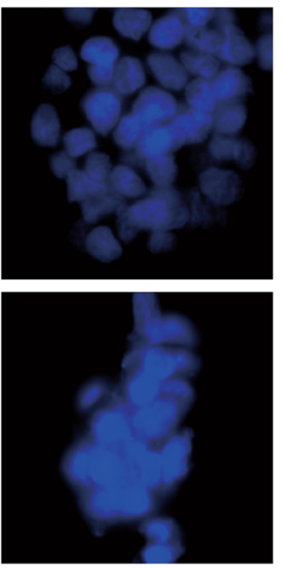

DAPI
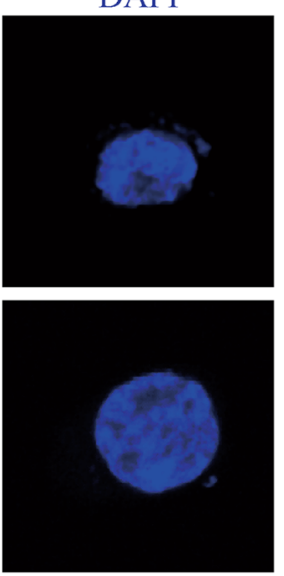

Merge
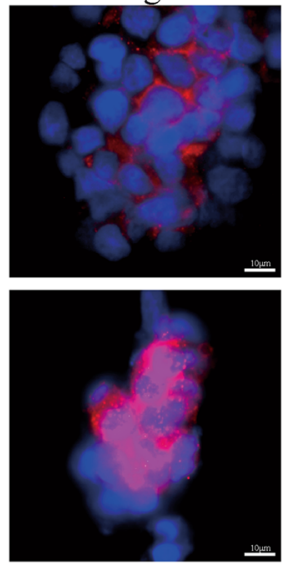

Merge
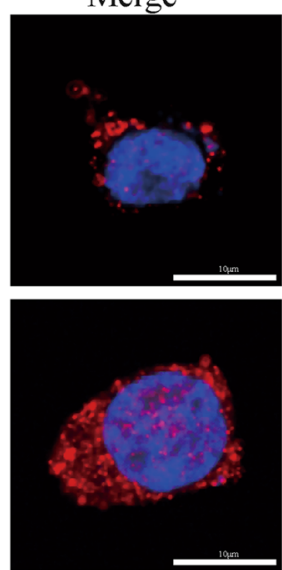

C

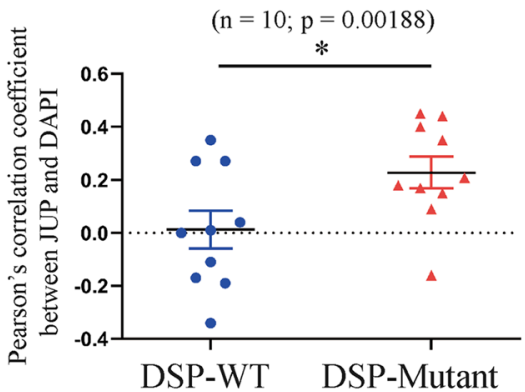

Fig. 5 Immunofluorescent staining examined JUP expression levels with either wild type or mutant DSP. HEK293T cells were transfected with either wild type or mutant plasmids. Blue indicate nuclear (DAPI) and red indicate JUP. a Representative images of the immunofluorescent staining of transfected HEK293T cells; b Confocal microscopic detection of the colocalization of JUP with DAPI; c Colocalization analysis of JUP and DAPI $(n=10)$. DSP, Desmoplakin; JUP, Junction plakoglobin; WT, wild type 
defects [20]. Non-specific heterozygous DSP-deficient mice demonstrate substantial adiposity and fibrosis in the ventricular myocardium, recapturing the human AC phenotype [21]. Nuclear translocation of the desmosomal protein plakoglobin (JUP) and suppression of $\mathrm{Wnt} / \beta$-catenin signaling pathway activity are found to be the underlying mechanism [21]. However, cardiac-restricted DSP-deficient mice develop a biventricular form of $\mathrm{AC}$ and no significant changes in JUP or $\beta$-catenin expression were detected [22], indicating that mechanisms other than Wnt pathway are responsible. In addition, silencing in HL-1 cells result in decreased expression and redistribution of the $N a_{v} 1.5$ protein and reduced sodium current [23], indicating an orchestra of canonical and non-canonical pathways synergically modulated the disease pathogenesis. Hence, immortal lymphoblastoid cell lines from the DSP c.832delG carriers and non-carriers in this family are established as to investigate the molecular pathogenesis. However, in our study no obvious DSP expression is detected by either western-blot or flow cytometry (data not shown), hindering the utilization of this cell line in downstream study. Therefore, plasmid carrying DSP c.832delG is constructed and transfected into HEK293T cells. Upregulation of JUP and downregulation of $\beta$-catenin in the nuclear suggest canonical Wnt/ $\beta$-catenin signaling pathway is likely to play a central role in the development of AC phenotype as previously reported [21]. However, HEK293T cells are unable to simulate the character of cardiomyocyte, hindering further studies on noncanonical pathways and cardiac phenotype.

Various cell models have been established to explore the potential effect of mutations [24]. Buccal mucosa cells from AC patients exhibit redistribution of desmosomes and gap junction protein, similar to those observed in heart [25]. However, in-depth phenotypic and mechanistic studies are not possible due to its distinct cellular features from cardiomyocytes. Patients-specific induced pluripotent stem cells (iPSc) derived cardiomyocytes contain the unique mutations and complete genetic background [26], thus providing us an ideal model to investigate the precise etiology and molecular mechanism. Moreover, the combination of iPSc and latest genome editing technology, such as CRISPR/ Cas9, has been succeeded in correcting LQT causal mutations and reversing phenotype $[27,28]$, promoting it as a promising approach towards precision medicine, and thereby should be introduced in our future study.

\section{Limitations}

In the current study, only HEK293T, a non-cardiac cell line, is utilized. Though human non-myocardial cell lines have been used as a cell model for investigating adhesive junction functions in AC [29], the effects of mutant DSP may differ in HEK293T cells from cardiomyocytes. Furthermore, non-cardiac cells are unable to reproduce the phenotype observed in human disease. Human iPSCs derived cardiomyocytes contain the unique genetic background of the patients and features of cardiac cells, hence they are robust tools to perform future studies and explore the mechanistic pathways. Transgenic animals, especially murine genetic knock-ins, are the most powerful and convincing models to investigate human inherited diseases, and also also be considered in the future studies.

\section{Conclusion}

We find the novel DSP c.832delG variant, which is likely causal in our AC family. CMR is a powerful alternative approach for the diagnosis of $\mathrm{AC}$ with high spatial and temporal resolution, especially in asymptomatic and echocardiogram negative patients. Future studies using patient-specific stem cells or animal models on the impact of the novel mutation, will be warranted to elucidate its pathogenesis of AC.

\section{Supplementary information}

Supplementary information accompanies this paper at https://doi.org/10. 1186/s12872-020-01369-5.

Additional file 1. Next generation sequencing results of the propand.

\section{Abbreviations}

AC: Arrhythmogenic cardiomyopathy; AKAP9: A-kinase anchoring protein 9; CMR: Cardiac magnetic resonance; DSP: Desmoplakin; ECG: Echocardiogram; JUP: Junction plakoglobin; NGS: Next generation sequencing; SCD: Sudden cardiac death

\section{Acknowledgements}

Not applicable.

\section{Authors' contributions}

$M X$ and $X L$ designed the study; $X L$ also enrolled patients, collected data, and was a major contributor in writing the manuscript; YM performed the cell experiments; QW, LW and ZH analyzed the data and prepared the manuscript; ZC also performed the cell experiments; $\mathrm{DH}$ analyzed the sequencing data and designed the cell experiments; JW also enrolled patients and prepared the manuscript; All authors have read, revised and approved the final version of this manuscript.

\section{Funding}

Provincial and Ministry Joint Major Projects of National Health Commission of China (WKJ-ZJ-1703 to Meixiang Xiang) supported the design of the study and the sequencing experiments; National Natural Science Foundation of China (81470384 to Meixiang Xiang) supported the cell experiments; National Natural Science Foundation of China (81870203 to Meixiang Xiang, 81670304 to Dan Hu) supported data analysis and interpretation; National Natural Science Foundation of China (81670259 to Meixiang Xiang) supported the writing of the manuscript.

Availability of data and materials

The datasets used and/or analyzed during the current study are available from the corresponding author on reasonable request.

Ethics approval and consent to participate

This study was approved by the ethics committee of Second Affiliated Hospital, Zhejiang University School of Medicine (2016-087). Written informed consent was properly obtained from all participants. 


\section{Competing interests}

The authors declare that they have no competing interests.

\begin{abstract}
Author details
'Department of Cardiology, the Second Affiliated Hospital, Zhejiang University School of Medicine, 88 Jiefang Road, Hangzhou 310009, Zhejiang, China. ${ }^{2}$ Department of Radiology, the Second Affiliated Hospital, Zhejiang University School of Medicine, 88 Jiefang Road, Hangzhou, Hangzhou 310009, Zhejiang, China. ${ }^{3}$ Experimental Teaching Center, School of Basic Medical Sciences, Zhejiang University, 866 Yuhangtang Road, Hangzhou 310058, Zhejiang, China. ${ }^{4}$ Department of Cardiology and Cardiovascular Research Institute, Renmin Hospital of Wuhan University, 238 Jiefang Road, Wuhan 430060, China. ${ }^{5}$ Provincial Key Lab of Cardiovascular Research, 88 Jiefang Road, Hangzhou 310009, Zhejiang, China.
\end{abstract}

Received: 19 August 2019 Accepted: 30 January 2020

Published online: 11 February 2020

\section{References}

1. Corrado D, Link MS, Calkins H. Arrhythmogenic right ventricular cardiomyopathy. N Engl J Med. 2017;376(1):61-72.

2. Thiene G, Nava A, Corrado D, Rossi L, Pennelli N. Right ventricular cardiomyopathy and sudden death in young people. N Engl J Med. 1988; 318(3):129-33.

3. Corrado D, Basso C, Pavei A, Michieli P, Schiavon M, Thiene G. Trends in sudden cardiovascular death in young competitive athletes after implementation of a preparticipation screening program. JAMA. 2006; 296(13):1593-601.

4. Marcus FI, McKenna WJ, Sherrill D, Basso C, Bauce B, Bluemke DA, Calkins H, Corrado D, Cox MG, Daubert JP, et al. Diagnosis of arrhythmogenic right ventricular cardiomyopathy/dysplasia: proposed modification of the task force criteria. Circulation. 2010;121(13):1533-41.

5. Corrado D, Wichter T, Link MS, Hauer R, Marchlinski F, Anastasakis A, Bauce B, Basso C, Brunckhorst C, Tsatsopoulou A, et al. Treatment of arrhythmogenic right ventricular cardiomyopathy/dysplasia: an international task force consensus statement. Eur Heart J. 2015;36(46):3227-37.

6. Marcus Fl, Edson S, Towbin JA. Genetics of arrhythmogenic right ventricular cardiomyopathy: a practical guide for physicians. J Am Coll Cardiol. 2013; 61(19):1945-8.

7. Norgett EE, Hatsell SJ, Carvajal-Huerta L, Cabezas JC, Common J, Purkis PE, Whittock N, Leigh IM, Stevens HP, Kelsell DP. Recessive mutation in desmoplakin disrupts desmoplakin-intermediate filament interactions and causes dilated cardiomyopathy, woolly hair and keratoderma. Hum Mol Genet. 2000;9(18):2761-6.

8. Kapplinger JD, Landstrom AP, Salisbury BA, Callis TE, Pollevick GD, Tester DJ, Cox MG, Bhuiyan Z, Bikker H, Wiesfeld AC, et al. Distinguishing arrhythmogenic right ventricular cardiomyopathy/dysplasia-associated mutations from background genetic noise. J Am Coll Cardiol. 2011;57(23): 2317-27.

9. Bhonsale A, Groeneweg JA, James CA, Dooijes D, Tichnell C, Jongbloed JD, Murray B, te Riele AS, van den Berg MP, Bikker $\mathrm{H}$, et al. Impact of genotype on clinical course in arrhythmogenic right ventricular dysplasia/cardiomyopathyassociated mutation carriers. Eur Heart J. 2015;36(14):847-55.

10. Castelletti S, Vischer AS, Syrris P, Crotti L, Spazzolini C, Ghidoni A, Parati G, Jenkins S, Kotta MC, McKenna WJ, et al. Desmoplakin missense and nonmissense mutations in arrhythmogenic right ventricular cardiomyopathy: genotype-phenotype correlation. Int J Cardiol. 2017;249:268-73.

11. Te Riele AS, Agullo-Pascual E, James CA, Leo-Macias A, Cerrone M, Zhang M, Lin X, Lin B, Sobreira NL, Amat-Alarcon N, et al. Multilevel analyses of SCN5A mutations in arrhythmogenic right ventricular dysplasia/cardiomyopathy suggest non-canonical mechanisms for disease pathogenesis. Cardiovasc Res. 2017;113(1):102-11.

12. Xiong Q, Cao Q, Zhou Q, Xie J, Shen Y, Wan R, Yu J, Yan S, Marian AJ, Hong K. Arrhythmogenic cardiomyopathy in a patient with a rare loss-of-function KCNQ1 mutation. J Am Heart Assoc. 2015:4(1):e001526.

13. Richards S, Aziz N, Bale S, Bick D, Das S, Gastier-Foster J, Grody WW, Hegde M, Lyon E, Spector E, et al. Standards and guidelines for the interpretation of sequence variants: a joint consensus recommendation of the American College of Medical Genetics and Genomics and the Association for Molecular Pathology. Genet Med. 2015;17(5):405-24.
14. Roberts B, Haupt A, Tucker A, Grancharova T, Arakaki J, Fuqua MA, Nelson A, Hookway C, Ludmann SA, Mueller IA, et al. Systematic gene tagging using CRISPR/Cas9 in human stem cells to illuminate cell organization. Mol Biol Cell. 2017;28(21):2854-74.

15. Puckelwartz M, McNally EM. Emery-Dreifuss muscular dystrophy. Handb Clin Neurol. 2011;101:155-66.

16. Gallicano Gl, Kouklis P, Bauer C, Yin M, Vasioukhin V, Degenstein L, Fuchs E. Desmoplakin is required early in development for assembly of desmosomes and cytoskeletal linkage. J Cell Biol. 1998;143(7):2009-22.

17. Lopez-Ayala JM, Gomez-Milanes I, Sanchez Munoz JJ, Ruiz-Espejo F, Ortiz M, Gonzalez-Carrillo J, Lopez-Cuenca D, Oliva-Sandoval MJ, Monserrat L, Valdes M, et al. Desmoplakin truncations and arrhythmogenic left ventricular cardiomyopathy: characterizing a phenotype. Europace. 2014;16(12):1838-46.

18. Rasmussen TB, Hansen J, Nissen PH, Palmfeldt J, Dalager S, Jensen UB, Kim WY, Heickendorff $L$, Molgaard $H$, Jensen $H K$, et al. Protein expression studies of desmoplakin mutations in cardiomyopathy patients reveal different molecular disease mechanisms. Clin Genet. 2013;84(1):20-30.

19. Fressart V, Duthoit G, Donal E, Probst V, Deharo JC, Chevalier P, Klug D, Dubourg $\mathrm{O}$, Delacretaz $\mathrm{E}$, Cosnay $\mathrm{P}$, et al. Desmosomal gene analysis in arrhythmogenic right ventricular dysplasia/cardiomyopathy: spectrum of mutations and clinical impact in practice. Europace. 2010;12(6):861-8.

20. Corrado D, Basso C, Judge DP. Arrhythmogenic cardiomyopathy. Circ Res. 2017;121(7):784-802.

21. Garcia-Gras E, Lombardi R, Giocondo MJ, Willerson JT, Schneider MD, Khoury DS, Marian AJ. Suppression of canonical Wnt/beta-catenin signaling by nuclear plakoglobin recapitulates phenotype of arrhythmogenic right ventricular cardiomyopathy. J Clin Invest. 2006;116(7):2012-21.

22. Lyon RC, Mezzano V, Wright AT, Pfeiffer E, Chuang J, Banares K, Castaneda A, Ouyang K, Cui L, Contu R, et al. Connexin defects underlie arrhythmogenic right ventricular cardiomyopathy in a novel mouse model. Hum Mol Genet. 2014;23(5):1134-50.

23. Zhang Q, Deng C, Rao F, Modi RM, Zhu J, Liu X, Mai L, Tan H, Yu X, Lin Q, et al. Silencing of desmoplakin decreases connexin43/Nav1.5 expression and sodium current in HL1 cardiomyocytes. Mol Med Rep. 2013;8(3):780-6.

24. Sommariva E, Stadiotti I, Perrucci GL, Tondo C, Pompilio G. Cell models of arrhythmogenic cardiomyopathy: advances and opportunities. Dis Model Mech. 2017:10(7):823-35

25. Asimaki A, Protonotarios A, James CA, Chelko SP, Tichnell C, Murray B, Tsatsopoulou A, Anastasakis A, Te Riele A, Kléber AG, et al. Characterizing the molecular pathology of Arrhythmogenic cardiomyopathy in patient Buccal mucosa cells. Circ Arrhythm Electrophysiol. 2016;9(2):e003688.

26. Yoshida Y, Yamanaka S. Induced pluripotent stem cells 10 years later: for cardiac applications. Circ Res. 2017;120(12):1958-68.

27. Yamamoto $Y$, Makiyama T, Harita T, Sasaki $K$, Wuriyanghai $Y$, Hayano M, Nishiuchi S, Kohjitani H, Hirose S, Chen J, et al. Allele-specific ablation rescues electrophysiological abnormalities in a human iPS cell model of long-QT syndrome with a CALM2 mutation. Hum Mol Genet. 2017;26(9): 1670-7.

28. Limpitikul WB, Dick IE, Tester DJ, Boczek NJ, Limphong P, Yang W, Choi MH, Babich J, DiSilvestre D, Kanter RJ, et al. A precision medicine approach to the Rescue of Function on malignant Calmodulinopathic long-QT syndrome. Circ Res. 2017;120(1):39-48.

29. Asimaki A, Syrris P, Wichter T, Matthias P, Saffitz JE, McKenna WJ. A novel dominant mutation in plakoglobin causes arrhythmogenic right ventricular cardiomyopathy. Am J Hum Genet. 2007:81(5):964-73.

\section{Publisher's Note}

Springer Nature remains neutral with regard to jurisdictional claims in published maps and institutional affiliations. 